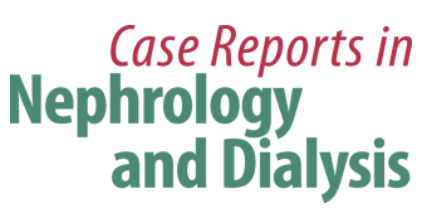

Case Rep Nephrol Dial 2016;6:96-100

\title{
Maintenance Hemodialysis Using Native Arteriovenous Fistula in a Patient with Severe Generalized Recessive Dystrophic Epidermolysis Bullosa
}

\author{
Takayasu Ito $^{a}$ Eiji Ishikawa $^{a}$ Hiroshi Matsuo $^{a}$ Mika Fujimoto $^{a}$ \\ Tomohiro Murata $^{a}$ Kenichi Isoda $^{\mathrm{b}}$ Hitoshi Mizutani $^{\mathrm{b}}$ Masaaki Ito $^{\mathrm{a}}$ \\ ${ }^{a}$ Department of Cardiology and Nephrology, Mie University Graduate School of Medicine, \\ Tsu, Japan; ${ }^{b}$ Department of Dermatology, Mie University Graduate School of Medicine, \\ Tsu, Japan
}

\section{Keywords}

Hemodialysis · Recessive dystrophic epidermolysis bullosa - Arteriovenous fistula · Chronic renal failure

\begin{abstract}
Renal failure and infectious disease are strongly associated with morbidity and mortality in patients with severe generalized recessive dystrophic epidermolysis bullosa (RDEB-sev gen). However, it is reportedly difficult to introduce hemodialysis with an arteriovenous fistula (AVF). We encountered a 32-year-old man with RDEB-sev gen in whom hemodialysis with a native AVF was introduced that favorably affected his long-term survival. This patient eventually died because of cachexia related to the recurrence of cutaneous squamous cell carcinoma 51 months after hemodialysis introduction. We believe that in this patient, the frequency of vascular access troubles related to infection or reduction of blood flow was probably low as a result of hemodialysis with his native AVF. Thus, it seems likely that patients with RDEB-sev gen with end stage kidney disease who are on hemodialysis can be successfully managed with a native AVF.




\section{Case Reports in Nephrology and Dialysis}

\section{Introduction}

Severe generalized recessive dystrophic epidermolysis bullosa (RDEB-sev gen) is an autosomal recessive disease with a poor prognosis that is characterized by generalized blistering of the skin and mucous membranes in response to slight mechanical stimulation. The main causes of death in patients with RDEB-sev gen are cutaneous squamous cell carcinoma, renal failure, infectious disease, and dilated cardiomyopathy [1-3]. Long-term prognosis is especially dependent upon complications such as renal failure and infectious disease [4]. When patients with RDEB-sev gen progress to end stage kidney disease (ESKD), hemodialysis with a permanent vascular catheter or peritoneal dialysis is introduced. However, it can be difficult to introduce hemodialysis with an arteriovenous fistula (AVF) because of easy blistering and vulnerability of the skin due to the need for repeated puncture of the AVF and the pressure required to ensure hemostasis after completion of a dialysis session. We herein present a patient with RDEB-sev gen in whom we introduced hemodialysis with a native AVF who survived 4 years and 3 months from the introduction of hemodialysis.

\section{Case Report}

A 32-year-old man with RDEB-sev gen was referred to our nephrology department because of proteinuria, hematuria, and renal dysfunction. He had generalized blistering of the skin on his birth. His clinical condition and pathological findings of skin biopsy led to a definitive diagnosis of RDEB-sev gen at 0 years of age. He had mitten deformities of his hands and feet, oral cavity blisters and scars, and low body weight $(28 \mathrm{~kg})$ at the initial visit to our nephrology department. His hemoglobin was $6.8 \mathrm{~g} / \mathrm{dl}$, and serum creatinine was $4.7 \mathrm{mg} / \mathrm{dl}$. Over the next 3 months, his serum creatinine level gradually increased to $6.5 \mathrm{mg} / \mathrm{dl}$. Although renal biopsy was not performed, end stage renal failure due to secondary amyloidosis was suspected with the finding of a high serum amyloid A level $(1,250 \mu \mathrm{g} / \mathrm{ml})$.

At the age of 32, native AVF anastomosis between the left median antebrachial vein and left radial artery was made for hemodialysis as the skin of his left elbow was dry and without blisters. Because uremia had developed and he and his family hoped for hemodialysis instead of peritoneal dialysis, single-needle hemodialysis was introduced. The needle was fixed with tape on a bandage, taking special care not to tape his skin directly (fig. 1). Astriction after removing the needle also took special care at the hemodialysis. In order not to create shear stress, we pressed the site to remove the needle vertically from directly above. These ways of fixing the needle and pressing the site to remove the needle had not formed any blisters in his puncture site. He received hemodialysis therapy for $3.5 \mathrm{~h}$ thrice weekly and his $\mathrm{Kt} / \mathrm{V}$ was more than 1.4. Maintenance hemodialysis was performed with native AVF for 21 months with no vascular access troubles related to infection or reduction in blood flow. Unfortunately, his native AVF was completely obstructed by thrombi after 21 months of hemodialysis. When percutaneous transluminal angioplasty failed, a permanent vascular catheter was selected for vascular access. Two cuffed, single-lumen catheters, Bio-Flex ${ }^{\mathrm{TM}}$ TesioR Cath (Medcomp, Harleysville, Pa., USA), were inserted into a subcutaneous tunnel under the left subclavian chest wall and placed in the superior vena cava (fig. 2). We selected his left subclavian vein for the puncture site. Neither of his internal jugular veins was appropriate for the puncture because his neck was very short and had severe erosion. Hemodialysis was then performed with a permanent vascular catheter for the next 30 months. When catheter thrombi prevented blood removal, urokinase was introduced into the catheter 30 
min prior to hemodialysis. When one catheter was obstructed, single-mode hemodialysis was performed using the other catheter. In the case of obstruction of both catheters due to thrombi, his permanent vascular catheter was replaced. This occurred three times. In one instance, we tried to insert a two-lumen catheter, the Split Stream ${ }^{\text {TM }}$ OTW Catheter (Medcomp). However, erosion at the catheter exit site worsened and long-term use of that catheter was difficult. Catheter-related infections were a frequent recurrence, and antibiotics were administered each time. Cutaneous squamous cell carcinoma was replaced eight times during the course of treatment. The patient died 51 months after hemodialysis introduction because of cachexia related to the recurrence of cutaneous squamous cell carcinoma.

\section{Discussion}

In the present case of a patient with RDEB-sev gen, we highlight two clinically important issues. First, because his hemodialysis was initiated with his native AVF, the frequency of vascular access-related infection or reduction of blood flow was reduced. To the best of our knowledge, this is the first report of a patient with RDEB-sev gen introduced to hemodialysis using a native AVF. In these types of patients, peritoneal dialysis or hemodialysis with a permanent vascular catheter for blood purification have previously been reported [5-8], but most reports discussed difficulty establishing AVF or arteriovenous graft [9]. Moreover, in these patients, significant problems with repeated punctures and maintenance of hemostasis of the shunt after the establishment of AVF persisted. It is very important not to create shear stress in the dermis during the puncture, adjustment of the needle, and while attempting to achieve hemostasis of the shunt. In our case, the frequency of vascular access troubles, such as infection or reduction of blood flow, was obviously low as a result of hemodialysis with a native AVF. Although hemodialysis with native AVF is not applicable to all patients with RDEB-sev gen, it can be of great benefit to appropriate candidates.

Second, long-term effective dialysis management of ESKD is possible in patients with RDEB-sev gen. Iida et al. [10] reported a patient with RDEB-sev gen who underwent hemodialysis with a permanent vascular catheter and survived for more than 4 years. Although the prognosis for patients with RDEB-sev gen who undergo dialysis therapy is still unclear, the mortality of RDEB-sev gen patients who do not undergo dialysis therapy is very high. Fine et al. [1] reported 5 of 141 patients with RDEB-sev gen who died due to ESKD, and the cumulative risk for death from renal failure was $12.3 \%$. The cause of death in our case was cachexia due to the recurrence of cutaneous squamous cell carcinoma. With respect to renal failure, as a result of maintenance hemodialysis with native AVF, and later a permanent vascular catheter, this patient survived for 51 months.

\section{Conclusion}

Maintenance hemodialysis with a native AVF can be introduced to patients with RDEBsev gen complicated by ESKD. Although it may not be initiated in all cases, infection related to the vascular access with a native AVF appeared to be less frequent than with a permanent vascular catheter. Hemodialysis with a native AVF could favorably affect the prognosis for patients with RDEB-sev gen and ESKD. 


\section{Statement of Ethics}

Due to the patient's death, we obtained informed consent from his parents.

\section{Disclosure Statement}

The authors have declared no conflicts of interest.

\section{References}

1 Fine JD, Johnson LB, Weiner M, Stein A, Cash S, DeLeoz J, Devries DT, Suchindran C; National Epidermolysis Bullosa Registry: Inherited epidermolysis bullosa and the risk of death from renal disease: experience of the National Epidermolysis Bullosa Registry. Am J Kidney Dis 2004;44:651-660.

2 Mann JF, Zeier M, Zilow E, Scharer K, Anton-Lamprecht I, Waldherr R, Andrassy K, Ritz E: The spectrum of renal involvement in epidermolysis bullosa dystrophica hereditaria: report of two cases. Am J Kidney Dis 1998;11:437-441.

3 Sidwell RV, Yates R, Atherton D: Dilated cardiomyopathy in dystrophic epidermolysis bullosa. Arch Dis Child 2000;83:59-63.

4 Suzuki H, Io H, Asanuma K, Takeda Y, Ohsawa I, Horikoshi S, Hasegawa T, Mizoguchi M, Ikeda S, Tomino Y: Successful maintenance hemodialysis with permanent vascular catheter in a patient with recessive dystrophic epidermolysis bullosa hereditaria (in Japanese). Toseki Kaishi 2009;42:899-904.

5 Chan SM, Dillon MJ, Duffy PG, Atherton DJ: Nephro-urological complications of epidermolysis bullosa in paediatric patients. Br J Dermatol 2007;156:143-147.

-6 Ahmadi J, Antaya R: Successful peritoneal dialysis in a patient with recessive dystrophic epidermolysis bullosa. Pediatr Dermatol 2007;24:589-590.

-7 Tammaro F, Calabrese R, Aceto G, Lospalluti L, Garofalo L, Bonifazi E, Piccolo T, Pannarale G, Penza R: End-stage renal disease secondary to IgA nephropathy in recessive dystrophic epidermolysis bullosa: a case report. Pediatr Nephrol 2008;23:141-144.

8 Sakan S, Basic-Jukic N, Tomasevic B, Kes P, Bandic Pavlovic D, Peric M: Vascular access for chronic hemodialysis in a patient with epidermolysis bullosa dystrophica Hallopeau Siemens. Hemodial Int 2013;17:126-129.

-9 Baloch MS, Fitzwilliams B, Mellerio J, Lakasing L, O'Sullivan G: Anaesthestic management of two difficult modes of delivery in patients with dystrophic epidermolysis bullosa. Int J Obstet Anesth 2008;17:153158.

10 Iida H, Hasegawa T, Okuma K, Io H, Tomino Y, Ikeda S: Successfully maintained hemodialysis for the treatment of chronic renal failure in a patient with Hallopeau-Siemens type recessive dystrophic epidermolysis bullosa. J Dermatol 2012;39:1088-1089. 


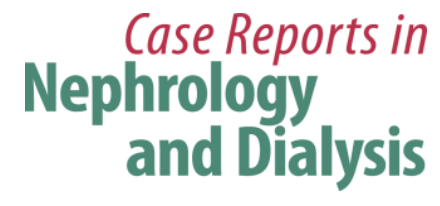

Case Rep Nephrol Dial 2016;6:96-100

(c) 2016 The Author(s). Published by S. Karger AG, Basel www.karger.com/cnd

Ito et al.: Maintenance Hemodialysis Using Native Arteriovenous Fistula in a Patient with Severe Generalized Recessive Dystrophic Epidermolysis Bullosa

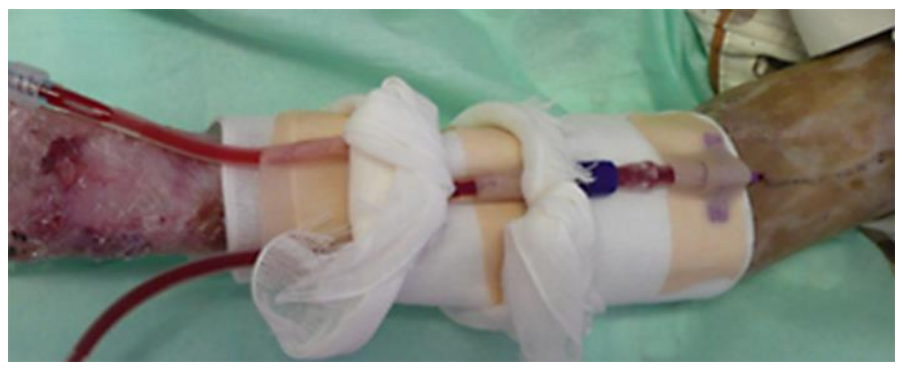

Fig. 1. Adjustment of the needle after puncture of the native AVF. Single-needle hemodialysis was performed by puncturing the native AVF. The needle was fixed with tape overlying a bandage.

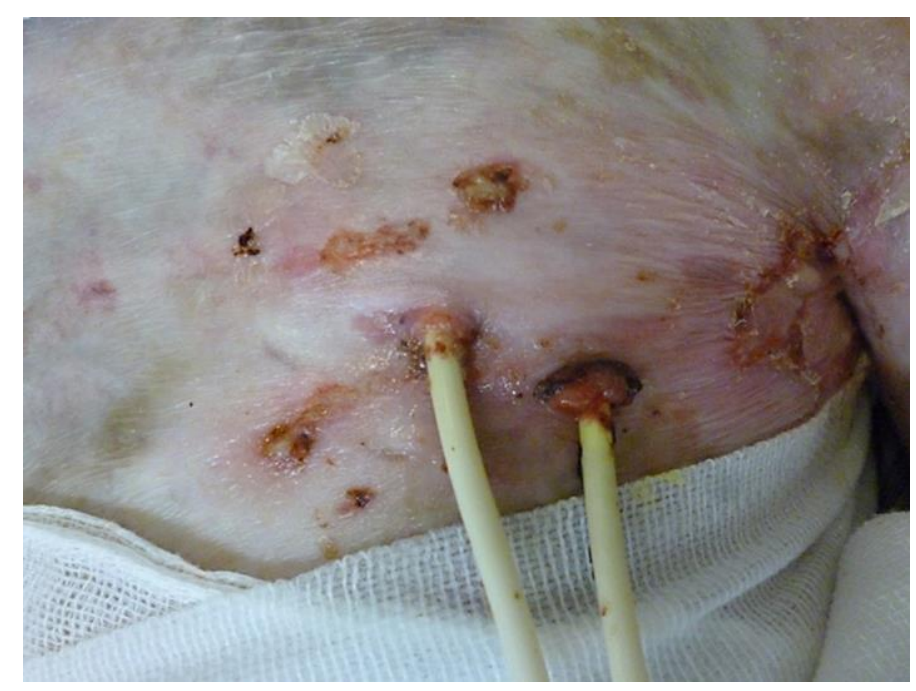

Fig. 2. Exit site of the permanent vascular catheter. Two cuffed, single-lumen catheters, Bio-Flex ${ }^{\mathrm{TM}}$ TesioR Cath (MedComp), were inserted into a subcutaneous tunnel under the left subclavian chest wall. 